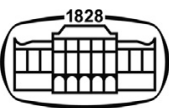

AKADÉMIAI KIADÓ

\section{Resolution and}

Discovery

$5(2020) 1,13-17$

DOI:

$10.1556 / 2051.2020 .00086$

(c) 2020 The Author(s)

\title{
HRTEM image-stitching for measurement of distances
}

\author{
GYÖRGY ZOLTÁN RADNÓCZI ${ }^{1 *} \bullet$, ZOLTÁN HERCEG ${ }^{2}$ and \\ TAMÁS RAFAEL KISS ${ }^{2}$
}

${ }^{1}$ Centre for Energy Research, Budapest, Hungary

2 Technoorg Linda Co. Ltd., Budapest, Hungary

Received: March 23, 2020 • Accepted: September 21, 2020

Published online: October 30, 2020

\section{ORIGINAL RESEARCH PAPER}

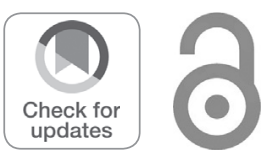

\begin{abstract}
Very accurate measurement of distances in the order of several $\mu \mathrm{m}$ is demonstrated on a single crystal Si sample by counting the lattice fringes on stitched high resolution TEM/STEM images. Stitching of TEM images commonly relies on correspondence points found in the image, however, the nearly perfect periodic nature of a lattice image renders such a procedure very unreliable. To overcome this difficulty artificial correspondence points are created on the sample using the electron beam. An accuracy better than $1 \%$ can be reached while measuring distances in the order of $1 \mu \mathrm{m}$. A detailed description of the process is provided, and its usability for accurately measuring large distances is discussed in detail.
\end{abstract}

\section{KEYWORDS}

HRTEM, calibration, epitaxy

\section{INTRODUCTION}

The evolution of materials science and device technology led to very accurate control of thin film growth and deposition rates. A need for direct measurement of film thicknesses and large distances is obvious, however, the accuracy of modern microscopes is usually around 1$2 \%$. This accuracy is only achieved if strict imaging requirements are met regarding sample height and defocus. This may be improved by the use of accurate calibration standards like the commonly known $\mathrm{Mag}^{\star} I^{\star} \mathrm{Cal}$ standards [1] but in some applications (e.g. the manufacturing of such calibration standards) more accurate measurements are desired.

Very accurate measurements can be carried out when the distances in the image are calibrated against a known lattice period. This way the accuracy will be better than one lattice period, however the distance is limited to the field of view. In practice this means $\sim 2 \AA$ accuracy over a distance of $40-80 \mathrm{~nm}=400-800 \AA$ which will result in a $<0.5 \%$ relative accuracy. As the field of view is limited to $40-80 \mathrm{~nm}$ for lattice images on most microscopes this method is limited to measuring distances fitting into this area. A few more limitations need to be observed: the lattice period must be known accurately (variable composition alloys, high strain and similar are not allowed) and the microscopes image distortion needs to be corrected if measurements/calibration is carried out in the outer regions of the image.

For measuring larger distances one needs to carry out multiple calibration steps to ensure accurate measurements on smaller magnifications where the lattice is not resolved. As each step has its own error accuracy is deteriorating with increasing distances and decreasing magnifications. In case the sample is single crystalline with a reasonably resolvable lattice over the distance to be measured one has the option to record multiple images and stitch them together and make an accurate calibration against the stitched lattice image.

Stitching of micrographs is a commonly used technique for detailed imaging of large areas [2], however the methods commonly used are not suitable for perfectly periodic images as crystal lattices. The main difficulty of this process is that a perfect lattice will not have easily

*Corresponding author. E-mail: radnoczi.gyorgy.zoltan@ energia.mta.hu 
identifiable correspondence points to be used for stitching. These are indeed needed, so artificial stitching marks must be created on the atomic scale. Fortunately modern transmission electron microscopes have condenser systems capable of producing very sharp electron beams which will burn through most materials upon exposures ranging from a few seconds to a few minutes depending on condenser system settings, sample material and thickness. A reasonably thin Si sample for HRTEM imaging requires 10-30 s exposure for a hole formation with spot size 3 (used for HRTEM imaging) and about 3 min with spot size 8 (used for STEM lattice imaging). As the sample is exposed to a sharp beam carbon contamination often builds up near the illuminated spot. These small carbon heaps as well as the holes burnt by the electron beam can be used as artificial stitching marks.

In this work the creation and use of such artificial stitching marks is described and an example on measuring a distance larger than one micron is presented.

\section{EXPERIMENTAL}

Thin sections of a $\mathrm{Si}$ based structure were prepared by conventional Ar ion milling [3] with $10 \mathrm{kV}$ accelerating voltage for rough milling and $3 \mathrm{kV}$ for final polishing. The structure consists of $\mathrm{Si}$ and $\mathrm{SiGe}$ layers of various thicknesses grown epitaxially onto a Si (100) wafer. The interfaces between the layers are accurately parallel with the (100) substrate surface, a uniformity of the layer thicknesses is assumed. The thin section for TEM imaging was prepared with $\{110\}$ surfaces constraining the electron beam near to $<110>$ direction within the crystal. Samples were imaged by a Thermo Scientific Themis 200 image corrected transmission electron microscope operated at $200 \mathrm{keV}$ and used in STEM mode. A standard double tilt sample holder allowed accurate alignment of the sample. Initially the thin sections were screened to find large areas that have the same crystallographic orientation throughout, and are reasonably uniform in thickness. The required uniformity in crystallographic orientation is unfortunately only found in thicker $(\sim 100 \mathrm{~nm})$ areas, however if the sample is reasonably well prepared (surfaces of the sample are nearly parallel) these areas will still be suitable for lattice resolution imaging over distances of a few $\mu \mathrm{m}$. It is important to note here that only the lattice period is needed for calibration purposes, hence low quality lattice images of thick, contaminated and somewhat misaligned areas are satisfactory.

Once the area was selected a series of overlapping images were recorded with uniform magnification. Before recording an image a correspondence point was created at the corner of the image as shown on the chart presented in Fig. 1a. The sequence for recording the images was as follows:

1. recording image $\mathrm{N}-1$ (with a freshly burnt hole at the corner towards the next image)

2. shifting the sample so that the last hole is still within the field of view near the opposite edge.
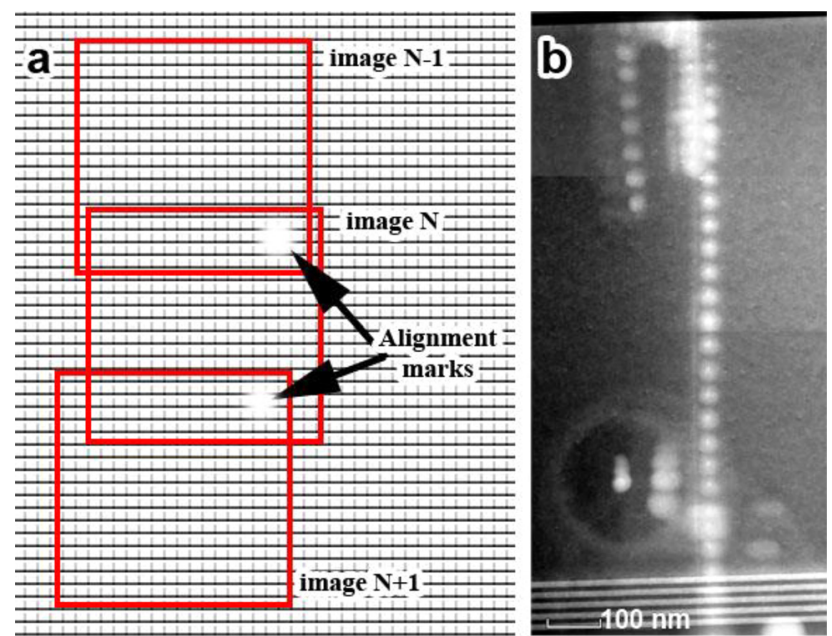

Fig. 1. (a) Schematic illustration of the overlapping image frames and alignment (stitching) marks described in the experimental section and (b) an overview image of the Si layer that was measured by our method. A series of stitching marks burnt in the sample throughout its entire thickness is also visible. These were the stitching marks used to align individual frames to each other

3. Burning a new hole with the electron beam

4. recording image $\mathrm{N}$

The imaging mode of the microscope can be freely selected provided that a reasonable lattice image is obtained.

\section{RESULTS AND DISCUSSION}

Images were recorded as described above along a line across a $1.2 \mu \mathrm{m}$ thick Si layer. The nominal magnification of the images is $1,963,000 \times$ and the resolution of the HAADF images is $1,024 \times 1,024$. The stitching marks are seen in an overview image shown in Fig. 1b. The first three high resolution images (starting from the top of the film) are presented in Fig. 2, with a detailed view on the alignment marks.

From the images we can conclude that the alignment marks are easily distinguished and seem suitable for accurate alignment. In our experience manual alignment is most often carried out with fair certainty, however, in some cases the uncertainty is about one or two lattice fringes. Here we note that only uncertainty along the surface normal causes error in the final thickness measurement, and this is estimated conservatively 2 fringes/image. In this case the lattice fringes i.e. rows of bright features parallel to the substrate surface are $1 / 2 \mathrm{a}_{\mathrm{Si}}=0.2716 \mathrm{~nm}$ apart corresponding to the $(002)_{\mathrm{Si}}$ maximum in the Fourier transform of the image.

The error of the measurement will depend mainly on this type of error, as the lattice dimensions are accurately known and counting lattice fringes should also be carried out without errors.

For this demonstration the images were manually aligned and the lattice fringes were counted manually. Of course many software applications and algorithms are 


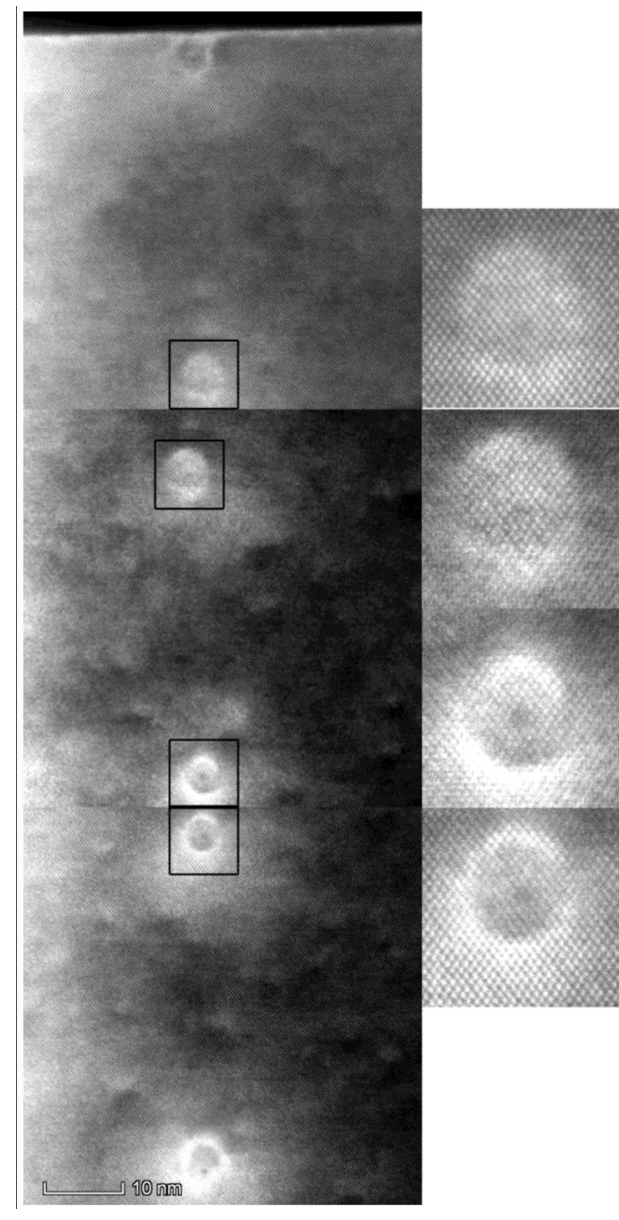

Fig. 2. Lattice images with stitching marks. The first 3 lattice images in the series are shown here with the stitching marks in detail. Stitching marks extend to an area about 10-20 lattice fringes with much smaller details making accurate alignments possible

available to carry out this alignment, and the lattice fringes are also easily counted on an intensity profile extracted from the stitched image. Finding the rough position of an alignment mark can be done by searching for extremes of the intensity on a blurred image (or quarter of the image as the alignment mark is surely within a specific quarter of the image). Then the neighborhood of this position can be used for alignment based on Normalized Cross-Correlation, (NCC). It is important, however, that large enough areas of the lattice image are included to guarantee proper matching of the lattice in the neighboring images.

Determining the number of the lattice fringes can be done either by fitting a cosine function on each image and calculating the number of periods between the two alignment marks or by extracting a relatively wide intensity profile from the stitched image and counting minima/ maxima with an appropriate algorithm.

In our case the automated alignment failed in some cases hence the results presented below are based on manual alignment.

The stitched image is not shown here as it is too large to include. Please refer to the Supplementary Material for this image. The image consists of 28 individual frames of which 26 are needed to cover the uppermost layer. The first interface from the top surface of the structure is at 3,758 lattice fringes distance corresponding to a layer thickness of $3,758^{\star} 0.2716 \mathrm{~nm}=1,020.6728 \mathrm{~nm}$ with an expected error of $\operatorname{sqrt}(25)^{\star} 0.5431 \mathrm{~nm}=2.72 \mathrm{~nm}$ and a $0.27 \%$ relative error. It is important to note here that some images had poor quality and needed noise filtering to make manual counting of the lattice fringes possible. The file provided in the Supplementary Material contains both the original and filtered images with the exactly same alignment as layers.

The result can be compared to the usual accuracy of the microscope magnification calibration which is around $2 \%$ for a modern microscope. Furthermore the stitched image will have a much higher resolution than a single image with the same field of view making more accurate measurements possible (depending on the size of the details that are used as endpoints in the distance measurements).

In case the sample is bent or strained the lattice image can be recorded with individual tilt correction for each frame. This way the original layer thickness (or any other distance within the single crystalline structure) can be measured rather than the actual physical distance influenced by sample bending or warping. As bending or warping of the thin sections is a common artefact of ion milling it is very important to note that the method presented here will not be influenced by this type of artefacts as long as their effect can be neglected within one lattice image frame.

As mentioned above the alignment marks are burnt into the sample by a focused electron beam. The process is slightly different for TEM and STEM modes. In STEM mode the beam is always focused and it can be arbitrarily positioned within the field of view. The user only needs to set the position and switch on the beam without scanning for a predetermined time. In TEM mode the user needs to focus the beam manually to the desired position with the condenser system. This will imply close inspection of a very bright spot on the fluorescent screen (if there is one), the use of proper eye protection is necessary. Furthermore condenser lens currents corresponding to focused beam are to be noted for reproducible beam formation and the position on the screen is to be marked (e.g. with the beamstop) where the alignment mark needs to be burnt.

In TEM mode the formation of the hole is clearly seen on the fluorescent screen (use proper eye protection while focusing the beam and observing hole formation!) and usually takes less than $20 \mathrm{~s}$ depending on beam settings. In STEM mode a longer time $(\sim 1-2 \mathrm{~min})$ is required as the beam current is usually lower for lattice resolution imaging. In this case only minimal sample drift $(<1 \mathrm{~nm} / \mathrm{min})$ is permitted as the hole will only form if the electron beam heats the same spot throughout the exposure. This requirement is rather hard to fulfill as the sample stage is moved after each image, causing transient drift. To compensate this we used the jog mode of the piezo stage available on our microscope, one should however avoid 
using the piezo stage for moving the sample over larger distances (e.g. between frames) as piezo actuators may have larger transient drift as the mechanical stage.

Minimal sample drift is advantageous though as it will make the shape of the alignment marks unique, helping to identify them if needed.

In both cases it is important that the sample is free from contamination (usually hidrocarbon/organic contamination may be present on the sample surface after ion milling or longer storage times). This type of contamination will brake down under the electron beam and form thick carbon heaps on both surfaces of the sample. A sample clean enough will withstand long exposures to focused electron beam without excessive carbon build-up. A perfectly clean sample will have a hole in it after proper exposure to the electron beam whereas a small heap of carbon will build up on the surface in case the sample is a little contaminated. Both holes and small carbon heaps are suitable for proper alignment. If the sample is heavily contaminated the carbon heap will be too wide and diffuse preventing precise alignment and proper imaging. Also a carbon ring forms around the illuminated area for HRTEM imaging which will appear on subsequent images as the sample is shifted. In our experience fresh FIB cut samples are usually clean enough but $\mathrm{Ar}$ ion milled samples sometimes need plasma cleaning for reasonable results.

The size of the alignment marks should be as small as possible for accurate alignment, in practice about 10 lattice fringes is ideal. Larger marks are more difficult to align accurately. Smaller holes or carbon heaps are difficult to form reliably, more importantly they might also change during imaging making the alignment less accurate.

Measuring large distances and recording image series implies moving the sample over relatively large distances compared to HRTEM frame size. Normally the sample height (defocus) and orientation is changing somewhat from frame to frame making adjustments sometimes necessary. One should keep in mind that no perfect HRTEM images are needed for this measurement, well defined lattice fringes and proper identification of the alignment marks are the only requirements which allow less frequent adjustments. As these adjustments are indeed time consuming one should strive to minimize the need for these.

The sample height may change due to sample stage misalignment/imperfections (usually not expected), inaccurate preparation of the sample, or sample bending. Special care should be taken to cut/prepare the thin section exactly perpendicular to the zone axis of the imaging (i.e. the crystallographic direction parallel with the electron beam as illustrated in Fig. 3). For Si sample thicknesses up to 80-100 $\mathrm{nm}$ will be acceptable for lattice imaging. The accuracy of the sample orientation should be within a few degrees to keep the sample within a reasonable defocus range. Bending of the sample also leads to defocus, but the more disturbing misalignment will have a larger impact on the imaging in case the sample is bending. Ideally bending should be less than $0.5^{\circ}$. Larger angles will call for excessive tilt correction involving also height correction which is very time

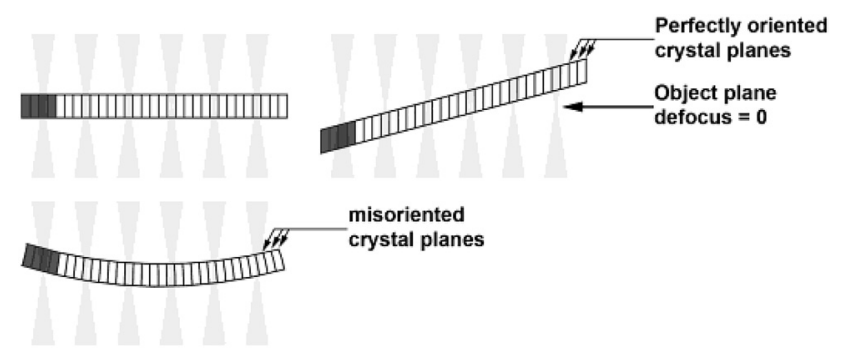

Fig. 3. Illustration of defocus and misalignment occurring on improperly prepared samples. If the sample is cut with inaccurate orientation (upper right) a defocus is experienced as the sample is moved. If the sample is bending (lower left) both defocus and misalignment will disturb imaging making time consuming adjustments necessary

consuming. As a rule of thumb one should select an area of the sample with mostly uniform orientation that has straight bending contours or no bending contours when aligned to zone axis.

The effect of sample defocus and misalignment will be different for STEM and HRTEM imaging modes. In TEM mode a wide range of the defocus is acceptable, as periodic images may be obtained with high defocus values, and perfect focusing conditions are hard to determine. The edge of the alignment mark may aid finding perfect focus. We also note here that the appearance of the alignment mark is substantially changed with defocus which may have an impact on the alignment accuracy. One should also keep in mind that the uncertainty in the defocus leads to an uncertainty in the contrast transfer function (CTF) meaning that only the periodicity of the image is preserved but the contrast may be inverted. This adds an uncertainty of half a lattice fringe distance to the alignment accuracy as keeping track of and taking into account CTF variations complicates the measurement unreasonably. In STEM-HAADF mode the perfect focusing conditions can be determined based on image resolution, and no contrast inversion is possible, hence the alignment accuracy is somewhat better as compared to HRTEM imaging, however, the STEM measurement is less tolerant to changes in sample height and deviations from the perfect alignment calling for more frequent focusing and realignment.

The error of the measurement depends partly on the accuracy of the alignment and number of alignments to be done. The accuracy of alignments and lattice imaging requires a reasonably high magnification whereas minimizing the number of necessary alignments calls for the largest possible field of view (the lowest reasonable magnification and highest available camera/recording resolution). The images shown in Fig. 2 were recorded with $1.963 \mathrm{M} \times$ magnification and a resolution of 1,024 by 1,024 in STEM mode. The spacing of the 002 lattice fringes in these images is 5.46 pixels. In our experience magnifications down to 480 $\mathrm{k} \times$ (TEM mode) are suitable for the measurement on our Thermo Scientific Themis 200 microscope with a $4 \mathrm{k}$ by $4 \mathrm{k}$ resolution. 
It is important that errors like image distortions, magnification errors, image drift does not impact the accuracy as long as the lattice fringes can be identified one by one and the alignment marks are fairly recognized. This means that the only requirement for imaging is the proper imaging of the lattice fringes in TEM or STEM mode, no previous calibration of the microscope is needed and the process is fairly tolerant against drift related (or other) distortions (mainly in STEM mode), and undefined lens aberrations or defocus in TEM mode.

\section{CONCLUSION}

A method for very accurate distance measurement was demonstrated with a typical error of $\sim 0.3 \%$ or $3 \mathrm{~nm}$ over a distance of $1 \mu \mathrm{m}$. The process relies on very small alignment marks burnt into the sample with the focused electron beam, hence the process changes the original structure during measurement. For this reason the method should be considered as partly destructive. The method described here may be used for accurately measuring thickness of an epitaxial or single crystalline layer of a known phase and for creating accurate calibration standards for TEM/STEM instruments.

\section{ACKNOWLEDGMENTS}

This work is supported also by the project VEKOP-2.3.3-152016-00002 of the European Structural and Investment Funds.

\section{SUPPLEMENTARY MATERIAL}

Electronic Supplementary Material (ESM) associated with this article can be found in the online version at https://doi. org/10.1556/2051.2020.00086.

\section{REFERENCES}

1. McCaffrey, J. P.; Baribeau, J. M. Microsc. Res. Tech. 1995, 32(5), 449-54. https://onlinelibrary.wiley.com/doi/10.1002/jemt.1070320507.

2. Chalfoun, J.; Majurski, M.; Blattner, T.; Bhadriraju, K.; Keyrouz, W.; Bajcsy, P.; Brady, M. Sci. Rep. 2017, 7, Article number: 4988. https:// www.nature.com/articles/s41598-017-04567-y.

3. Barna, Á.; Pécz, B.; Menyhard, M. Micron 1999, 30(3), 267-76. https://www.sciencedirect.com/science/article/pii/ S0968432899000116 